\title{
DU SACRÉ DUEL AU SACRÉ DÉBATTU: LA LÉGITIMITÉ EN ÉCHO DES SOUVERAINS MAGHRÉBINS
}

\author{
JOCELYNE DAKHLIA \\ E. H. E. S. S., París
}

La difficulté, et peut-être même le malaise avec lesquels les chercheurs peuvent aujourd'hui aborder la question du pouvoir sacré dans l'Islam sont sans doute plus importants dans le cas du Maghreb que dans des contextes historiques orientaux. Orient ou Occident de l'Islam, les cadres généraux du problème sont bien connus, et ils renvoient à la fois à un vide historiographique, un «blanc» de la recherche, et à des vérités d'évidence héritées de la tradition dite "orientaliste». Bien que l'Islam soit remarquablement absent, ou peu s'en faut, des recherches classiques sur la royauté sacrée - de Frazer à Marc Bloch ${ }^{1}$ - la notion de royauté sacrée est en effet, de manière paradoxale, une référence acquise dans les premières recherches marquantes sur les dynasties musulmanes, à travers, notamment, l'idée de la théocratie abbasside ${ }^{2}$.

La coupure historiographique que l'on vient d'évoquer, l'isolement

1 Rappelons que M. Bloch, par exemple, ne cite le cas de l'Islam qu'à deux reprises, dans Les Rois thaumaturges, dont une occurrence en note. Il évoque la croyance que le sang du calife, mais aussi de "tous les Koraïchites" guérit de la rage. Voir rééd. Paris, 1983, 84-85. Les travaux récents des historiens de l'Europe confirment ou même accentuent cette coupure avec les études sur le monde musulman, absent de toute démarche comparatiste. Voir par exemple A. Guéry qui recourt à des comparaisons avec le cas du Japon ou de la Chine, par exemple, mais ignore l'Islam. Cf «La dualité de toutes les monarchies et la monarchie chrétienne», in A. Boureau et Cl.-S. Ingerflom (eds.), La Royauté sacrée dans le monde chrétien, Paris, 1992. La vision stéréotypée du politique dans l'Islam que perpétue la tradition d'une séparation des "études orientales" peut être résumée par ces deux motifs: "intrication du religieux et du politique" et "absence de l'individu", soit par ces quelques lignes de L. Dumont, qui viennent significativement comme une simple note, dans un appendice à Homo Hierarchicus: "Le cas de l'Islam fait difficulté à première vue, comme une religion individualiste qui n'a pas donné l'autonomie au domaine politique. Mais l'Islam n'a pas (préparé et) accepté, comme le christianisme, la révolution moderne des valeurs; ce n'est pas une religion de l'individu au sens présent", "Nationalisme et communalisme», n. 5, 379, Appendice D, Homo hierarchicus, Paris, Gallimard, 1966.

2 Voir notamment les travaux classiques de I. Goldziher, E. Tyan, A. Abel, G. von Grunebaum. 
théorique encore sensible de toute réflexion sur l'Islam à ce propos, sont d'autant plus remarquables que c'est sur le motif de l'«emprunt" au monde non musulman que reposaient fondamentalement l'étude des formes de sacralisation du califat ou du sultanat; le scandale théorique et théologique de la sacralisation d'un homme -le Calife-successeur d'un homme -le Prophète- n'a en effet été longtemps résolu par les historiens que par l'argument de l'influence étrangère et de l'emprunt ou de l'imitation. Les études de I. Goldziher par exemple, comme celles, plus tardives, de M. Canard ou de D. Sourdel conçoivent au premier chef la sacralité du pouvoir politique en Islam comme un héritage direct des Sassanides ou de Byzance ${ }^{3}$; la référence implicite ou explicite au césaro-papisme ou à la lutte du Sacerdoce et de l'Empire est aussi une ligne de force de ces approches ${ }^{4}$. Il s'ensuit un constant effet d'extranéité de la royauté sacrée, ou de la royauté tout court, qui alimente jusque dans des travaux historiques récents de faux débats sur l'authenticité et la fidélité à l'esprit de l'Islam.

Cette question est donc tout entière enserrée dans une problématique de l'essence de l'Islam et de sa spécificité politique, sans même que l'on songe à formuler dans son contexte cette question par ailleurs élémentaire et banale - peut-être vaine-: soit l'on admet qu'il est du sacré par nature dans toute forme de souveraineté ou de royauté ${ }^{5}$; soit l'on conçoit comme un type distinct les formes de pouvoir politique centrées sur la sacralisation du souverain.

La notion de «type», à cet égard, est au moins implicitement présente dans le cas des recherches sur le Maghreb, moins manifestement marqué par l'imitation de Byzance ou des Sassanides. Les faits d'emprunt, pourtant sensibles -quoique dans d'autres références ${ }^{6}-$ y sont

3 Voir les conclusions de Canard, M., "Le cérémonial fatimide et le cérémonial byzantin: essai de comparaison", Byzantion, xxi, (1951), 355-420, et D. Sourdel, «Questions de cérémonial abbasside", R. E. I., (1960), 121-148.

4 Voir par exemple Goldziher, I., Etudes sur la tradition islamique, extraites du Tome II des Muhammedanische Studien, trad. L. Bercher, Paris, 1984 ( $1^{\text {ère }}$ éd. 1952), 84 sq.

5 Voir «La royauté sacrée. Eléments pour un débat", introduction générale de J. Revel à A. Boureau et Cl.-S. Ingerflom (éds.), op. cit., 7-17. Voir Evans-Pritchard: "A mon avis, la royauté, en tous temps et en tous lieux, a été, dans une certaine mesure, une charge sacrée. (...) Je ne veux pas prétendre que, parce que toute royauté possède certaines caractéristiques de la royauté divine, la royauté divine ne constitue pas un type d'institution distinct par référence à ses autres caractéristiques", p. 94. "La royauté divine chez les Shilluk du Soudan nilotique" (1948), in Les anthropologues face à la religion et à l'histoire, Paris, 1974, 73-96.

6 Voir notamment les influences catalanes qui s'exercent sur les souverains maghrébins. Cf. Dufourcq, Ch. E., L'Espagne catalane et le Maghrib aux XIII e et XIV'e siècles, $\mathrm{Pa}-$ ris, 1966. 
presque éludés, à l'inverse, dans l'historiographie, surtout la plus récente. De fait le caractère globalement plus restreint, au Maghreb, des formes de sacralisation du politique explique sans doute une plus forte réceptivité des historiens de ces sociétés à une problématique de l'authenticité et de la pureté des modèles de pouvoir ${ }^{7}$.

Pour échapper, sans les occulter, à ces débats sur l'allochtonie des modèles et l'opposition des types, il est peut-être alors opportun de cesser de dissocier a priori des formules politiques antinomiques, afin de les envisager dans leur cohabitation et dans leur tension, tant au cours de leur élaboration que dans leur réception historiographique. $\mathrm{La}$ question, souvent indécidable, de la présence ou de l'absence de formes sacralisantes du pouvoir a par ailleurs fréquemment eu pour effet d'éliminer celle de la croyance et des formes de consensus politique qui s'établissent, ou échouent à s'établir, autour d'un modèle de pouvoir. Partant d'exemples maghrébins, je voudrais ainsi, de manière encore programmatique, m'interroger sur la simultanéité, dans un même contexte politique, de plusieurs "types» de légitimation du pouvoir, et suggérer une plus fine focalisation de la recherche sur des faits de réception, sur des formes d'accord mais aussi de désaccord qui se manifestent autour de la production d'un modèle de gouvernement, et qui mettent en question son efficace ${ }^{8}$. Il s'agit par là de réagir contre des interprétations qui tendent constamment à «lisser" l'idéologie royale ou sultanienne, soit par un excessif monothétisme, soit en privilégiant à l'excès, dans une sorte d'irénisme, l'idée de réceptions consensuelles et communielles dans lesquelles chacun -souverain, Cour, sujets...- trouverait ses marques et son compte.

\section{STYLES, SCÈNES ET TYPES}

A examiner le problème de la sacralité politique depuis l'Occident musulman, on est dans un premier temps fortement et peut-être légiti-

7 Voir par exemple Laroui, A., Islam et modernité, Paris, 1987.

8 Cf. Revel, J., op. cit. Ce travail voudrait se situer dans une continuité et dans le dialogue avec celui de Kably, M., Société, pouvoir et religion au Maroc à la fin du Moyen-âge, Paris, 1986 ou de Bourquia, R., State and Rural Society in Morocco: the Zemmour and Zayan Confederation in the 19th and 20th Centuries, $\mathrm{Ph} \mathrm{D}$, Université de Manchester, 1987; "L'Etat et la gestion du symbolique au Maroc précolonial", in R. Bourquia et N. Hopkins (éds.), Le Maghreb: approches des mécanismes d'articulation, Casablanca, 1991. 
mement enclin à défendre la thèse d'un pluralisme et plus encore d'un dualisme des modèles historiques, l'idée de "types" politiques distincts, indépendamment de l'échelle d'étendue et de puissance des "royaumes" pris en compte dans la comparaison ${ }^{9}$. La tradition ou les traditions politiques maghrébines privilégient en effet, dans la longue durée, des formes très fortement paritaires et égalitaires de la présence du souverain parmi ses sujets, indissociables d'une exigence de visibilité 10 . Cette contrainte de parité exclut notamment, au moins sur l'échelle longue du temps, la coutume de la prosternation, ou encore celle du souverain "caché», recevant en audience derrière un voile ou un rideau, sitr $^{11}$. L'égalitarisme maghrébin, l'exigence que le souverain n'oublie en aucun cas qu'il est un homme semblable aux autres, sont souvent poussés si loin que l'humilité et l'absence de "dépassement" deviennent ostentatoires; les princes du Maghreb aiment ainsi à adopter une forme d'anonymat, se font "oublier" pour se fondre dans une assemblée de lettrés, d'étudiants ou de poètes. C'est dans la référence à un tel idéal qu'un faqih marocain, au début du XIX ${ }^{\mathrm{e}}$ siècle, relate sa rencontre avec Mawlāy Sulaymān:

«Il tira sa montre de sa poche pour la régler, et je vis qu'il l'attachait avec un mejdoûl de laine. A la prière du 'așr qu'il vint faire avec nous, je vis qu'il portait un pantalon rapiécé» ${ }^{12}$.

Le même faqìh évoque par ailleurs la modestie des repas de Mawlāy Sulaymān, qui n'est pas sans rappeler le pieux exemple de 'Umar b. 'Abd al-'Azīz, dans une référence non pas spécifiquement maghrébine, mais renvoyant à une centralité de l'Islam ${ }^{13}$.

Jusqu'au milieu du $\mathrm{xIx}^{\mathrm{e}}$ siècle, comme en témoigne le ministrechroniqueur Ibn Abī Dịāâ, la prétention à l'anonymat est également

${ }^{9}$ Cf. Dakhlia, J. et Valensi, L., "Le spectacle de la Cour: éléments de comparaison des modes de souveraineté au Maghreb et dans l'Empire ottoman", in G. Veinstein (éd.), Soliman le Magnifique et son temps, Paris, 1992, 145-157.

10 Voir Lefort, $\mathrm{Cl}$., "Hanna Arendt et la question du politique», Essais sur le politique, $X I X^{e}-X X^{e}$ siècles, Paris, 1986,65 sq.

11 Cf. Sourdel par exemple; Bazin, J., "Le roi sans visage», confirmé par des traditions orales in L'oubli de la cité, Paris, 1990.

12 Nāșirī, Kitāb al-Istiqșā, vol. X, Archives marocaines, 100. calife.

Voir par exemple les passages de la chronique de Tabarī se rapportant à ce même 
fréquente chez les Beys de Tunis, y compris de la part d'Aḥmad Bey, qui, par ailleurs, se fait construire un palais à l'imitation de Versailles ${ }^{14}$ :

«Plus d'une fois, il vint à l'improviste à la Mosquée en dehors des heures de la prière et s'asseyait derrière le cercle des étudiants et ni le professeur ni aucun des étudiants ne se levait à son approche» 15 .

Le même prince se mêle tout aussi «anonymement» à la foule lors de la célèbration du Mawlid, notamment:

«Il avait l'habitude de passer la nuit du Mouled à Tunis, au Dar al Bey, de faire la prière du soir à la Grande Mosquée puis de circuler à pied comme le commun des mortels à travers les rues et les souks de la ville, se laissant presser par le flot des piétons comme il les pressait luimêmes» ${ }^{16}$.

Or, cette simplicité, et plus encore cette volonté de se fondre ostensiblement dans la masse des sujets, sont expressément conçues dans la référence antithétique à un autre modèle de gouvernement, oriental celui-là et, pour la période envisagée, principalement ottoman. Conseillant à son maître de parcourir à cheval, entre une haie de troupes, la distance du Bardo à la Grande Mosquée, Ibn Abī Ḍiyāf se voit ainsi répondre:

«Le Sultan agit ainsi, par égard pour lui, nous ne devons pas l'imiter» ${ }^{17}$.

Sous le prétexte d'un respect du suzerain -de plus en plus formel et théorique à cette époque-c'est bien la dualité de deux paradigmes qui est en réalité en cause. On la retrouve formulée plus nettement encore dans le contexte marocain, pourtant plus ouvertement en concurrence idéologique avec le modèle ottoman 18 .

14 Le palais d'al Muhammadiyya. Cf. Brown, L. C., The Tunisia of Ahmed Bey, Princeton, 1975 .

15 Abdesselem, A., Ithäf ahl az-Zamān. Chronique du règne d'Ahmad Bey. Tunis, 1976, Chap. VI, p. 65.

16 Op. cit., 51

17 Op. cit., 50.

18 Voir El Moudden, A., Sharifs and Padishahs: Moroccan-Ottoman Relations from the 16th through the 18th Centuries. Contibution to the Study of a Diplomatic Culture, Ph. D, Princeton, 1992; "Les relations maroco-Ottomanes: quelques grands traits d'une culture diplomatique», in A. El Moudden (éd.), Le Maghreb à l'époque ottomane, Rabat, 1995, 13-19. 
Ainsi, comme d'autres ambassadeurs maghrébins, Tamgrūtī, par exemple, au $\mathrm{xv}^{\mathrm{e}}$ siècle, atteste la surprise des Marocains devant l'extrême sens de la hiérarchie qui prévaut parmi les Turcs; reçu par le Sultan à Istanbul, il s'étonne des préséances propres aux ministres euxmêmes:

«Jamais aucun d'eux ne doit traiter d'égal à égal avec son supérieur, soit en marchant au même rang, soit en portant un turban ou des vêtements de même qualité, soit en s'asseyant sur un siège semblable. Je n'ai jamais vu d'hommes observant les préséances plus scrupuleusement» ${ }^{19}$.

$\mathrm{Au} \mathrm{XIX}{ }^{\mathrm{e}}$ siècle encore, comme l'atteste Erckmann, par exemple, sous le règne de Mawlāy Hasan, un mélange d'austérité et d'égalitarisme -plus ou moins affectés-prévaut à la "cour» marocaine:

«Les palais impériaux sont construits en pisé et tout le luxe est réservé pour l'intérieur" 20.

Les audiences au palais se font aussi dans la simulation du dénuement:

«Des gens sont accroupis le long des murs, y compris les caïds. Dans ce milieu les caïds des provinces ne brillent pas; ils sont habillés le plus simplement possible et parlent peu car les murs peuvent avoir des oreilles» 21.

La contrainte de silence ne doit donc rien, dans ce cas, en théorie au moins, à l'étiquette. Le sultan lui aussi se différencie à peine de son entourage:

«Il s'habille en blanc, avec la plus grande simplicité, a les pieds nus dans des babouches et ne se distingue des autres Arabes que parce qu'il met son capuchon de laine sur la tête» 22 .

Mawlāy Hasan confirme en second lieu une tradition maghrébine du sultan à cheval, constamment en campagne ou sur les routes, pacifiant le royaume ou à l'écoute de ses sujets, dans la reproduction de cet-

19 Tamagrūtī, En-Nafhat el-Miskiya, trad. H. de Castries, Paris, 1929, 61.

20 Ibidem, 218-219.

21 Id.

22 Ibidem, 216. 
te même mise en scène de l'humilité ou de l'austérité ${ }^{23}$. En campagne, toujours selon le témoignage d'Erckmann, le mot d'ordre sultanien est celui de la visibilité et de la simplicité de la mise en scène:

"Le travail du makhzen se pratique toujours de la même manière: le grand vizir, entouré d'une douzaine de secrétaires très lettrés, siège généralement en plein air; il est assis sur un tapis, derrière une petite caisse en bois qui renferme quelques papiers.

Cette installation manque de prestige; on a peine à croire qu'on ait devant soi le Conseil supérieur de l'empire» 24.

L'austérité du palais s'expliquait cependant aussi comme une forme de reproduction de l'itinérance ou du gouvernement «à cheval»: le sultan y siège fréquemment en plein air, sous une tente, sous un pavillon mobile, dans un jardin, ou encore, "le dimanche», dans une écurie ${ }^{25}$. Or, à ce stade de la description, l'idée d'un dualisme des modèles est déjà fragile et discutable. Les souverains abbassides, par exemple, recevaient communément sous un pavillon, tarima; l'idée du palais comme reproduction du camp est également familière à l'Orient, de même que l'on retrouve, dans le contexte de l'empire de Soliman, par exemple, de longues descriptions de la "ville de toile» que constitue le camp itinérant ${ }^{26}$. Si l'on peut concevoir une dualité de types de gouvernement, celle-ci doit donc aussi tenir compte d'une communauté de lexique, qui inciterait à plutôt prendre en compte des inflexions: le pouvoir du camp est plus accentué pour le pôle maghrébin, par opposition au pouvoir du palais, plus prononcé dans la tradition longue de l'Orient. On pourrait encore, dans une oppossition inflexive, distinguer entre un pôle occidental qui privilégie le souverain exposé aux regards, et un pôle

${ }^{23}$ Sur les modalités maghrébines de la mehalla, voir Afif, M., "Les harka-s hassaniennes d'après l'oeuvre d' A. Ibn Zidane», Hespéris-Tamuda, 19 (1980-81), 153-168 Nordman, D., "Les expéditions de Moulay Hassan. Essai statistique», Id., 123-152; El Moudden, A., "Etat et société rurale à travers la harka au Maroc du XIX ${ }^{\mathrm{e}}$ siècle», The Maghreb Review (1983), 141-145; Dakhlia, J., "Dans la mouvance du prince: la symbolique du pouvoir itinérant au Maghreb», Annales ESC (1988), 735-760; Michel, N., "L'approvisionnement de la mhalla au Maroc au XIX ${ }^{\mathrm{e}}$ siècle", Hespéris-Tamuda (1991), 313340, et "Itinéraires de la mhalla (1757-1900)", Revue Maroc-Europe, n. 7 (1994), 81-115. Voir pour la période médiévale Ferhat, H. et Sebti, A., Al-Madina fí l-așr al-wasit, Beyrouth, 1994, 72-79.

24 Op. cit., p. 222.

25 Id., p. 224.

26 Voir Sourdel, D., op. cit., qui cite notamment, d'après Mas'ūdī, l'exemple d'un palais lakhmide conçu sur le plan d'une armée au combat. 
oriental où domine le souverain caché. De manière plus forte et conclusive, on distinguerait entre un pôle de la "royauté contractuelle», tant la rhétorique de la bay'a joue un rôle central dans la vie politique maghrébine, et une "royauté sacrée» ou "sacralisée» à l'autre extrémité de l'espace méditerranéen ${ }^{27}$.

Ces oppositions demeurent pertinentes dans la mesure même où elles sont revendiquées dans le contexte local, indigène. Pour suivre encore le même témoignage, Erckmann atteste cette dualité: "Les Marocains, dit-il, accusent les Turcs de se christianiser et disent que le Sultan de Stamboul n'est pas chérif»" 28.

Il est vrai qu'en d'autres occurrences le gouvernement ottoman est, à l'inverse, érigé en modèle positif, mais toujours sur le mode antithétique. Essayant d'apaiser une révolte des habitants de Fès, le sultan marocain écrit ainsi à ses sujets en colère:

«L'Ottoman réside à Constantinople et ses ordres sont exécutés à Tlemsen, dans l'Inde et dans l'Yemen. Les populations de ces régions ne l'ont jamais vu, et cependant elles se soumettent aux ordres de Dieu. Ô vous qui croyez, obéissez à Dieu, son Prophète et celui d'entre vous qui vous gouverne» 29 .

La référence au prince «invisible» se conclut donc malgré tout par le rappel que le souverain n'est que l'un des membres de la communaté des croyants.

Légitime et sans doute heuristique dans une certaine mesure, la distinction de deux catégories de modèles politiques -sacralisés ou nonn'en apparaît pas moins comme une clasification trop rigide. D'une part, on le constate, ces deux types ne sont cités que dans la référence l'un à l'autre, comme deux modalités d'une même réalité. D'autre part, les exemples historiques dont nous disposons, par delà le discours classificatoire, révèlent plutôt, selon des formules politiques éminemment variables, une combinaison des deux types.

27 On sait la prégnance actuelle de ce modèle du contrat au Maroc. J. Bazin montre le caractère illusoire de cette opposition, puisque le roi «sacré» est sacré par contrat (ouvrage en cours). Voir L. Dumont sur la royauté contractuelle in "La conception de la royauté dans l'Inde ancienne", Appendice C, Homo hierarchicus..., 351-375.

28 Erckmann, J., Le Maroc moderne, París, 1885, p. 222.

29 Nāșirī, t. X, 1907 op. cit., p. 59. 


\section{UNE COMBINATOIRE ET DEUX PÓLES}

En premier lieu, les formes de sacralisation les plus manifestes -couronne, prosternation...- ne sont pas absentes des cours maghrébines, quoique, comme l'a souligné R. Brunschvig, sur un mode extrêmement instable et restreint ${ }^{30}$. Inversement, l'on a été sensible de longue date aux traits d'humilité, effective ou de façade, qui caractériseraient certains califes orientaux, indépendamment du respect qui est plus classiquement affiché envers les représentants de la Loi ${ }^{31}$. De même retrouve-t-on, dans le contexte ottoman, notamment, le jeu de rôles qui pousse le sultan à se faire aussi artisan ou poète ${ }^{32}$.

Plus encore, on peut interpréter la recherche de l'ubiquité qui est propre aux souverains maghrébins, ou à nombre d'entre eux, comme une forme de sacralisation. Non seulement elle renvoie à une forme de perfection, mais elle se fonde sur une hybridité qui est le propre de la créature d'exception. Dans la seule description -éminemment banaled'un homme, le sultan, qui combine jusque dans son costume et ses postures, la perfection du sage, du lettré et du guerrier, se trouve une forme de "scandale logique» et de miracle -sur un modèle clairement muhammadien ${ }^{33}$.

L'ostentation de la simplicité n'est d'ailleurs en rien incompatible avec les signes de l'exception, et notamment avec ces formes de délégation du geste ou de la parole qui désignent le corps du souverain comme une sorte de corps vide ${ }^{34}$. Ainsi, étonné qu'il était par l'extrême modestie de la cour marocaine, Erckmann n'en soulignait pas moins des pratiques de ce type, contredisant en apparence la simplicité de mise:

30 Brunschvig, R., La Berbérie orientale sous les Hafsides, Tome II, Paris, 1947, chapitre VIII, rarement souligné par les historiens postérieurs.

31 Le cheikh-ül-islam notamment dans le cas ottoman.

32 Avec l'adoption d'un nom de plume, d'une autre identité, qui, à ma connaisance au moins, n'est pas coutumière au Maghreb.

33 Voir al-'Umarī, Masālik al-Abșär fi Mamālik al-Amsāar, trad. M. Gaudefroy-Demombynes, Paris, 1927, pp. 202-203 et p. 217.

34 Voir par exemple, pour le cas des Fatimides, M. Canard, op. cit., et P. Sanders, Ritual, Politics and the City in Fatimid Cairo, State University of New York, 1994; "From Court Ceremony to Urban Language: Ceremonial in Fatimid Cairo anf Fustāt», in Essays in Honor of Bernard Lewis, Princeton 1989, 311-321. 
«En public (le sultan) ne parle généralement que par l'intermédiaire du maître des cérémonies, qui répète ses paroles à haute voix, afin que nul n'en ignore» 35 .

De même le luxe des emblèmes sultaniens -parasol, lance, caparaçon...- contraste-t-il avec l'austérité du costume royal. On pourraît encore citer la présence dans les palais maghrébins de ces bêtes fauves -généralement des lions- qui symbolisent de manière classique dans les traditions islamiques la capacité du prince à inspirer l'effroi, hayba, et un effroi sacré, en même temps que son aptitude à domestiquer, à l'instar de Salomon, la création animale ${ }^{36}$. Erckmann s'est montré également sensible à cette familiarité du sultan avec les fauves:

«Des panthères apprivoisées promènent dans la cour du palais leurs pas souples et silencieux.

Nous avons vu souvent ces animaux sauter sur la tente aplatie où siège le conseil et s'y étirer pendant des heures» ${ }^{37}$.

Plus révélatrice encore d'une foncière indistinction des types, on retrouve sans surprise au Maghreb une classique correspondance entre la prospérité du royaume et le corps du souverain, et tout d'abord une immédiate relation entre son accession légitime au trône et l'harmonie du royaume. Cette relation est tout particulièrement marquée par les chroniqueurs lors de l'accession au trône d'un nouveau souverain, qui, et en particulier dans le cas marocain, doit souvent de surcroît surmonter le chaos de l'interrègne ${ }^{38}$. De manière stéréotypée, leurs narrations évoquent la baisse du prix des grains, ou celle du prix de la poudre à fusil, l'abondance des pluies et des récoltes.

On retrouve tout aussi classiquement dans les sources une relation entre la virilité du sultan et la fécondité du pays. Des femmes et des enfants présentent ainsi au souverain marocain, lors de ses mehallas, des

35 Op. cit., p. 216. Voir Bazin, J., «Princes désarmés, corps dangereux. Les rois-femmes de la région de Segu", Cahiers d'Etudes Africaines, 111-112 (1988), 375-441.

${ }^{36}$ Sur cette notion, voir Mottahedeh, R., "Justice, Kingship and the Shape of Society", Loyalty and Leadership in an early Islamic Society, Princeton, 1980; Dakhlia, J., "Sous le vocable de Salomon. L'exercice de la "justice retenue au Maghreb", Annales islamologiques, XXVII (1993), 169-180.

37 Op. cit., p. 224

38 Cf. Sebti, A., "Les crises dans la chronique dynastique marocaine», Cahiers d'Etudes Africaines, 119, (1990), 237-249, et Jamous, R. (qui à cet égard se réfère à Hocart), Honneur et baraka, Cambridge-MSH, 1981. 
jarres de lait, afin qu'il y plonge son doigt "pour la baraka» ${ }^{39}$. Les malheurs du royaume ne vont pas jusqu'à atteindre physiquement le roi, comme dans le cas de la royauté scythe, analysé par F. Hartog, mais le sultan est rendu responsable dans sa virilité de tous les troubles du royaume, et notamment des disettes et sécheresses ${ }^{40}$. Des traditions tunisiennes associent directement ces calamités au "saroual» des gouvernants politiques ${ }^{41}$. Monchicourt, au début du $\mathrm{xx}^{\mathrm{e}}$ siècle, rapporte qu'il se dit d'un caïd ou de tout autre personnage de pouvoir, que son saroual est "salé», en cas de sécheresse, "pur» dans le cas de pluies bénéfiques ${ }^{42}$. Aḥmad Bey aurait lui-même reconnu cette relation de correspondance entre le corps du roi et le corps du royaume:

«Le prince, apprenant un jour que Gafsa venait d'être grêlée, décida d'exempter des impôts de l'année les jardins de l'oasis. Etonnement de la cour. Protestation respectueuse du vizir. Le Bey expliqua alors confidentiellement qu'il se reconnaissait comme responsable du désastre parce qu'à ce moment même il était en conversation coupable avec la femme d'un de ses officiers" 43 .

Constater que les fondements du pouvoir souverain ne sont en rien différents au Maghreb ou dans l'Islam de ce qu'il sont dans le reste du monde n'est sans doute pas un aussi mince constat qu'il y paraît, tant domine un postulat de spécificité pour tout ce qui concerne le politique dans l'Islam. Il serait pourtant d'un intérêt limité de s'en tenir sur ce point au présupposé vague qui est celui de L. Dumont, par exemple, ou d'autres auteurs après lui, qui veut qu'un "fond" de croyance ou de magie, une sacralité diffuse, soient inhérents à l'institution royale ellemême ${ }^{44}$.

39 Weisgerber, F., Au seuil du Maroc moderne, Rabat, 1947, 93.

40 Voir Pseudo Jāhiz, Kitāb al-Tāj, ed. Zaki Pasha, Le Caire, 1914 trad. franç. Ch. Pellat, 1954, sur l'abstention à l'égard des femmes en situation de crise ou de guerre. Autres mentions dans la chronique de Seghir b. Yūsuf. Voir F. Hartog, Le Miroir d'Hérodote, Paris, 1980, sur l'idée que les malheurs du royaume affectent le corps du roi, et Evans-Pritchard, op. cit., sur l'idée que le roi peut être tué en situation de crise pour le royaume.

${ }^{41}$ Sur cette relation au Maroc, voir A. Dialmy, article à paraître dans Annales, HSS et $\mathrm{S}$. Gubert, recherche en cours.

42 Monchicourt, "Moeurs indigènes: les rogations pour la pluie (thlôb en nô)", Revue tunisienne (1915), 65-81; A. Bel, XIV Congrès des Orientalistes, 1905.

43 Monchicourt, id., p. 66.

44 Cf. Dumont, L., op. cit:; Guéry, A., "L'Oeuvre royale. Du roi magicien au roi technicien", Le Débat, n. 74 (mars-avril, 1993), 123-142. Voir "Kingship and the kings", 
Ou peut tout d'abord souligner que les deux "styles» de pouvoir politique qui ont été définis, antithétiques mais pas incompatibles, puisent de manière égale et légitime dans la tradition scripturaire islamique ${ }^{45}$. Sans que les limites de cet exposé permettent de développer ce point, on rappellera que le genre du hadith, notamment, fournit abondamment, en images de majesté et de royauté "sacrée», l'imaginaire politique, à travers notamment le motif du trône, 'arsh, qui peut être le trône divin ou salomonien ${ }^{46}$. Les récits prophétiques se rapportant à David et surtout à Salomon, la théologie du $M i^{c}$ râj confèrent aux images du mulk une inscription dans l'Islam infiniment plus forte et légitime que ne le donne à penser l'accent que l'on a porté de manière trop exclusive sur les emprunts à Byzance ou à la Perse sassanide.

Dans un autre registre, celui de la pratique politique, ces deux styles de souveraineté se rejoignent sur une base de "lois fondamentales" communes, et notamment sur la même contrainte de "justice» par exemple, qu'elle soit théologiquement rapportée à l'Islam ou définie sur un mode universel et a-islamique ${ }^{47}$. Sans doute deux formes de présence du souverain parmi ses sujets sont-elles alors actualisées: dans un contexte historique maghrébin, le sultan ou le personnage -pacha, bey ou dey- qui en tient lieu, est généralement contraint d'être physiquement présent à l'audience de justice réservée; pour les dynastes ou califes orientaux, la délégation de ce devoir est plus fréquente ou constitue la règle. Pourtant, même dans ce dernier cas, comme l'atteste la célèbre fenêtre ottomane au dessus du Divan, la présence invisible du prince est au moins suggérée, comme le soulignait, au $\mathrm{xv}^{\mathrm{e}}$ siècle, Guillaume Postel ${ }^{48}$.

Présent comme Dieu, d'une présence invisible, ou présent comme un homme, exposé aux regards et aux armes... cette alternative reproduit un débat juridique médiéval, comme l'a montré E. Kantorowicz, qui oppose le modèle du roi combattant parmi ses troupes et celui du "Grand Roi de Suse ou d'Ecbatane», caché dans son palais, mais

\footnotetext{
History and Anthropology, 1989. Ces faiblesses sont soulignées par A. Boureau et J. Revel dans l'ouvrage collectif cité supra.

45 Qui elle même se nourrit aussi de ces modèles de royauté. Thème plus amplement développé dans un ouvrage en cours de rédaction.

46 Cf. EI, ${ }^{\circ}$ arsh.

47 Voir notamment les travaux d'A. Lambton et en particulier «Justice in the Medieval Persian Theory of Kingship", Studia Islamica, XVII (1962) 91-119.

48 Necipoğlu, G., Architecture, Ceremonial and Power. The Topkapi Palace in the fifteenth and sixteenth centuries, MIT Press, 1991. Voir Postel, G., La République des Turcs.
} 
capable de tout voir et tout entendre dans son royaume ${ }^{49}$. L'auteur des Deux corps $d u$ roi citait à ce propos un traité pseudo-aristotélicien, traduit en latin au XIII ${ }^{\mathrm{e}}$ siècle, qui explorait les termes de ce débat, et qui évoque l'immense réception, dans les sociétés musulmanes, de ce véritable genre des «lettres d'Aristote à Alexandre» 50. L'une de ces «lettres» fournit ainsi une formulation aiguë du modèle de l'ubiquité, en ne le rattachant en apparence qu'à des référents royaux et orientaux:

"La cour de Cambyse, de Xerxès, de Darius, présentaient bien à leurs peuples l'image de la grandeur et de la majesté du prince; mais le prince lui-même résidait à Suse ou à Ecbatane, invisible, retiré dans un palais magnifique, brillant d'or, d'ambre et d'ivoire. De longues avenues se succédant les unes aux autres, offraient de stades en stades des enceintes superbes, où l'on n'entrait que par des portes d'airain. Hors de ces enceintes étaient établis par ordre les seigneurs les plus éminents Des soldats, attachés à la personne du roi, faisaient le service de l'intérieur. D'autres, faisant garde à chacune des entrées, recevaient les avis, prêtaient l'oreille à tout; de sorte que le roi lui-même, portant les noms de maître absolu et même de Dieu, voyait tout, entendait tout (...) Il y avait des courriers, des observateurs, des porteurs de messages, des gardes, des inspecteurs de signaux. L'ordre était tel, surtout parmi ces derniers, que, par le moyen de feux allumés de loin en loin, le roi savait le même jour, à Suse et à Ecbatane, ce qui était arrivé dans toute l'Asie.

Mais il y a autant de différence entre le Dieu qui gouverne le monde et le grand roi, qu'il y en a entre le grand roi et le plus vil et le plus faible des animaux" 51 .

La constitution d'un puissant réseau d'espions, qui rend possible l'invisible présence du souverain dans son royaume, constitue une recommandation classique des manuels de conseil aux princes ${ }^{52}$. Le thème s'en trouve également illustré à l'infini par le lieu commun du calife, ou du prince, qui sort à la nuit, incognito, pour entendre ce qui se dit de l'ordre du royaume. Or, ce motif de l'incognito du prince, ou de

49 Cf. Kantorowicz, Les deux corps du roi. Paris, 1989 (1ère éd. Princeton, 1957).

50 Sur le problème de ces matrices communes et de ces "lieux communs", voir Dakhlia, J., "La question des lieux communs. Des modèles de souveraineté dans l'Islam méditerranéen", in B. Lepetit (Ed.), Les Formes de l'expérience, Paris, 1995, 39-61. Cf. Kantorowicz, op. cit., 192, n. 216.

51 "Lettre d'Aristote à Alexandre sur le monde", annexe à La Politique, trad. Champagne, Paris, Charpentier, 1843 (référence communiquée par P. Pellegrin).

52 Voir par exemple "Nizam al Mulk; Traité de gouvernement", C, trad. Schefer, réed. Paris, 1984; Sourdel-Thomine, J., "Conseils du shaykh al Harawi à un prince ayyūbide». 
son ubiquité, se retrouve aussi, en un sens, derrière l'image de ce bey pouvant surgir à tout moment dans la Grande Mosquée, pour y entendre une leçon. On trouverait de la même façon, dans les régions occidentales de l'Islam, et indépendamment de la littérature sultanienne, fortement stéréotypée, l'idée que le prince juste doit recourir aux espions.

$\mathrm{Au}$ XVIII ${ }^{\mathrm{e}}$, Mawlāy Ismāî̉l, par exemple, souverain peu caché s'il en est, recourait, à travers sa légende au moins, à la même forme d'omniprésence invisible, grâce à un gigantesque réseau d'informateurs, si l'on en croit, tout au moins, Joseph de Leon:

"Afin d'informer le roi de tout ce qui se passait dans ses états, les pachas et caïds devaient entretenir à la Cour des agents chargés de lui faire savoir les nouvelles des provinces; ces hommes devaient aussi transmettre la correspondance échangée entre le souverain et leurs maitres. (...) Chaque agent devait quotidiennement lui présenter un rapport sur ce qui se passait dans sa province, soit en public, soit en privé, suivant l'importance des faits. Et si, par ses espions, le roi apprenait qu'on lui avait caché quelque chose, il punissait le pacha ou le caïd responsable, et l'agent qui n'avait pas transmis un ordre donné était passible de la peine capitale» 53 .

A plusieurs reprises, Joseph de Leon insiste sur l'efficacité du réseau d'informateurs et d'espions de Mawlāy Ismā'îl, mais d'une façon qui dénote moins l'observation directe que la rumeur indigène. Un idéal du pouvoir fort est en réalité à l'oeuvre dans son récit, fondé sur le contrôle d'un roi-dieu. Il est irréaliste en effet, même compte tenu de la légendaire dureté de Mawlāy Ismā'il, d'entendre à la lettre cette description, par exemple:

«Le moindre fonctionnaire était surveillé en secret et tout ce qu'il faisait était rapporté au roi qui pouvait alors exercer sa sévérité suivant la gravité du délit. Si les espions étaient convaincus de faux rapport, c'étaient eux qui perdaient la vie; s'il arrivait qu'ils fussent assassinés par ceux qu'ils étaient chargés de surveiller, ces derniers étaient à leur tour exécutés" 54 .

Un paradigme du souverain invisible est donc bien présent aussi au Maghreb. A l'inverse, dans les traditions plus centrales de l'Islam, l'in-

53 De la Véronne, Ch., Vie de Moulay Isma'ïl, roi de Fès et de Maroc, d'après Joseph de Leon (1708-1728), Paris, 1972, 51, et fo 171.

$54 \quad$ Id. 52. 
sistance continue des ouvrages de conseil aux princes sur la nécessité d'entretenir un réseau d'espions, a pour effet de désacraliser le souverain, par l'énoncé d'une simple règle de bon sens politique.

Il en est de même de cette recommandation politique classique, selon laquelle le prince doit préserver son "mystère» en établissant sa résidence hors de la capitale, évitant une trop grande familiarité avec ses sujets. G. Necipoğlu a notamment souligné ce point à propos de Topkap1, citant l'historien Bidlisi ${ }^{55}$. Cependant, la répétition même de cette argumentation du secret, dans des ouvrages qui, par ailleurs se diluent dans la vaste mouvance de l'adab-dans une culture "moyenne» et parfois même populaire, de l'honnête homme ${ }^{56}$ - cette constante réitération de la nécessité du mystère a sans doute en soi pour effet d'en démonter la teneur. Nombre de témoignages confèrent d'ailleurs une toute autre rationalité à cette pratique de la séparation du palais, ou de la ville princière, et de la capitale. Tabarī affirme ainsi que la raison de la construction de Samarra est que al-Ma'mūn tenait à disperser ses troupes pour empêcher qu'elles soient trop nombreuses dans la capitale 57 $\mathrm{Al}-$ Mas'ūdī évoque à cet égard la volonté du calife de protéger les habitants de sa capitale des brutalités de la soldatesque ${ }^{58}$. Al-Khațib alBaghdādī, enfin, reproduit la fiction d'un dialogue, à Baghdād, entre alManșūr et un ambassadeur byzantin, qui constitue une réfutation de l'argument du mystère. Le Grec dit en effet: «(...) Tes sujets demeurent avec toi dans ton palais, et lorsque les sujets sont avec le roi dans son habitation, son secret est divulgué»; al-Manșūr lui répond alors qu'il n'a pas de secret pour ses sujets 59 .

Sans être absolument fictifs ou caduques, les arguments référant au mystère sont donc constamment contrapposés à des arguments inverses, référant quant à eux à la pleine humanité du sultan. C'est pourquoi il convient d'analyser de manière plus dynamique, et dans une relation

55 Op. cit., p. 14 sq. Voir Barrucand, M., Urbanisme princier en Islam, Paris, 1985.

56 Comme l'atteste la très large diffusion d'une oeuvre de compilation telle que le Mustatraf d'al-Ibshīhī, par exemple. L'idée du caractère purement élitaire et clos de la culture du miroir est défendue, par exemple, par J. Sourdel dans «L'expression symbolique de l'autorité dans l'art islamique", La notion d'autorité au Moyen Age, Colloque international de la Napoule, 1978, Paris, 273-286.

57 Tome 6, Les Abbassides, 179.

58 Op. cit. t. IV, $1145, \S 2801$.

59 Al-Khațīb al-Baghdādì, L'introduction topographique à l'histoire de Baghdād, éd. G. Salmon, Paris, 1904. 
de tension, la simultanéité, dans un même contexte historique, d'une sacralisation et d'une désacralisation du souverain.

\section{L'ENVERS DU DÉCOR}

Quand bien même une tradition "sacralisante» prédomine en Orient et une tradition "humanisante» dans le Gharb, chacune admet aussi en partie, comme on vient de le voir, son propre envers. Le thème de «l'autre côté du décor» est d'ailleurs explicitement développé dans nombre de chroniques ou d'ouvrages d'édification et de divertissement. Goldziher a notamment cité ce motif à travers l'histoire d'al-Mutawakkil qui, après avoir fêté la Rupture du Jeûne, en recevant l'hommage de milliers de personnes, se couvre la tête de poussière, à son retour au palais, afin de s'humilier devant Dieu ${ }^{60}$. On peut également mentionner cette fable longuement rapportée dans le Mustatraf d'al-Ibshīhī: un roi reçoit ses sujets en audience un jour par semaine et vit le reste du temps dans une masure communiquant avec le palais, vêtu de laine, tressant des corbeilles pour gagner son pain ${ }^{61}$. La pompe et le mystère sont ainsi clairement démontrés comme un jeu d'illusion.

La construction d'une majesté politique sacralisée apparait d'autre part liée à une tension dans le temps. L'étude déjà mentionnée de G. Necipoğlu sur le cérémonial ottoman montre ainsi un processus de transition d'une situation de rapport "paritaire" entre le sultan et ses officiers, dans laquelle le souverain apparaît journellement à ses troupes et partage leur nourriture, vers une forme de pouvoir centré sur l'invisi-

60 Op. cit., 68.

61 "Lorsque le souverain eut terminé les affaires de son peuple, il se leva de son siège, prit par la main l'homme au nuage et l'emmena avec lui dans (l'intérieur de) son palais; il lui fit traverser le vestibule, le long duquel notre homme ne rencontra pas même un seul serviteur, et le roi le conduisit jusqu'à ce qu'ils parvinrent à une porte construite avec des branches de palmier, fixée à une vieille masure et à des murs qui menaçaient ruines, et en dedans de laquelle se trouvait une chambre délabrée avec un paillasson. Il n'y avait pas là pour dix dirhams d'objets; on n'y voyait qu'un mauvais tapis de prières, un baquet pour les ablutions, une natte en lambeaux, et quelques feuilles de palmiers. Le roi se débarrassa de ses vêtements royaux, endossa de mauvais habits de laine, se mit sur la tête un bonnet en crin (...)", pp. 473-476, chap. XXXI, cité dans la trad. Rat. Le modèle de référence est celui de David et de Salomon. Cf. Tha 'labī, Qișas al-Anbiyä', Tabarī. Voir Y. Knappert, Islamic Legends, Leiden, t. 1, 1985, «Salomon and his servants". 
bilité du Sultan et son retrait ${ }^{62}$. Or, lors d'une succession politique incertaine, l'un des fils de Mehmet II, Bayezid, doit revenir temporairement à l'ancien cérémonial, afin d'assurer son emprise sur les troupes ${ }^{63}$.

Le degré de "sacralisation" ou de fiction sacralisante du pouvoir serait donc fontion d'un rapport de forces politique, qui suppose que la position du sultan soit suffisamment sûre, affermie, pour qu'il puisse s'autoriser d'évidents "dépassements» de son mandat de simple gestionnaire de la communauté; ce n'est que dans une telle situation de force que ces marques d'exception peuvent à leur tour conforter sa prééminence et augmenter son pouvoir. Cette vision conditionnelle du problème contredirait notamment l'idée formulée par Evans-Pritchard que le roi est d'autant plus "sacré» que son pouvoir politique effectif est faible ${ }^{64}$.

L'exemple du rideau d'audience confirme assez cette dynamique, sans remettre en question l'idée de styles d'autorité différents. Non seulement la coutume du voile, sitr ou hijäb -sacralisant à la fois parce qu'il renvoie au dieu caché, à l'interdit de la figuration du Prophète et à l'indétermination sexuelle du souverain ${ }^{65}$ - non seulement cet usage est demeuré proscrit au Maghreb, mais la simple sédentarité du souverain provoque son impopularité. Le voyageur oriental al-'Umarī, par exemple, atteste cette exigence de confrontation avec le sultan, en Tunisie, au XIV siècle, sous le règne du hafside Abū Bakr b. Yahyā:

"C'est un fait qui agite les langues des Tunisiens que leur sultan actuel se dérobe aux regards, contrairement à la coutume de tous ses ancêtres; qu'il est peu soucieux d'examiner les affaires de ses officiers et de

62 Op. cit., 18-22. La tradition que le «souverain" goûte journellement le pain se perpétue dans les Régences de Tunis et d'Alger, mais elle est également attestée au Maroc. Voir Ch. de La Véronne, op. cit., pour l'exemple de Mawlāy Ismāeil, 48 et fo 157.

63 Id.

64 «(...) Je suggèrerais que c'est une institution typique, bien qu'elle n'en soit sans doute pas l'apanage, des sociétés à système lignager dominant, dans lesquelles les segments politiques constituent les éléments d'une structure vaguement organisée sans fonctions gouvernementales. Dans les sociétés de ce type, l'organisation politiqe revêt une forme rituelle ou symbolique qui dans les régimens ayant un dégré supérieur d'organisation cède le pas, bien que jamais totalement, à une administration centralisée», op. cit., 95.

${ }_{65}$ Voir Bazin, J., op. cit.; Miller, D. A., «Royauté et ambiguïté sexuelle», Annales ESC, (main-juin 1971), n. 3, 639-652; Dakhlia, J., "L'histoire est dans l'attente», Cahiers d'Etudes Africaines 119, (1990), 251-278. 
ses sujets, et qu'il est tout entier à ses plaisirs, malgré tout son courage, sa vaillance et ses bons sentiments» 66 .

Cinq siècles plus tard, une réaction identique est celle des Tunisiens lorsqu'Ahmad Bey commence la construction du palais d'al Muhammadiyya, comme en témoigne Ibn Abī Ḍiyāf:

"Le souverain fut alors l'objet de vives critiques car l'on disait qu'en s'isolant dans cette nouvelle résidence, en se livrant à l'oisiveté, il négligeait de siéger pour rendre la justice comme le faisaient ses prédécesseurs» 67 .

$\mathrm{Au}$ Maroc, le célèbre épisode de la rencontre d'al-Manșūr et d'Aḥmad Bābā de Tombouctou atteste plus nettement encore l'echec - au moins partiel-du sultan à reculer les limites de ce qui est admis en manière de dépassement de l'humanité ordinaire ${ }^{68}$ :

«Lorsqu' après avoir été rendu à la liberté, Ahmed Bāba se présenta au palais de al-Mansour, il remarqua que ce prince restait caché (selon la tradition des califes abbāssides et les souverains qui les imitèrent) derrière un rideau flottant qui le séparait du plublic, quand il donnait audience:

Dieu, qu'il soit exalté, dit alors le cheikh, a déclaré dans le Coran qu'aucun être humain ne pouvait communiquer avec Dieu autrement que par le révélation ou en demeurant caché derrière un voile; vous imitez donc le Maître des maitres; mais si vous avez à me parler, venez vers moi et écartez ce rideau» ${ }^{69}$.

Si le voile au Maghreb échoue à s'imposer, sa tradition en Orient n'est elle même pas si univoque, dans le sens de la sacralisation. Indépendamment des récusations dont elle peut faire l'objet de la part de théologiens, sur la base d'arguments semblables à ceux d'Aḥmad Bābā, les textes qui la préconisent en révèlent des significations ambiguës, des usages contradictoires, tour à tour sacralisants et désacralisants. Le $\mathrm{Ki}$ tâb al-Tāj, l'un des premiers et des principaux ouvrages de conseil aux princes qu'ait connu le monde musulman, est à cet égard exemplaire, et l'on notera d'emblée que ce texte a servi de matrice à d'innombrables

66 Op. cit., 118.

67 Op. cit., 65.

68 Sur cet épisode, voir Kaddouri, A. (Ed.), Mahdisme. Crise et changement dans l'histoire du Maroc, Rabat, 1994.

69 Nāṣirī, Kitāb al-Istiqṣā, Archives marocaines, vol. XXXIV, 1936, 226. 
compilations ultérieures, et a également servi de source à des auteurs tels que Mas'ū $\bar{d} i$ ou TTabarī ${ }^{70}$. Or, les pages ou les chapitres que l'auteur du Livre de la Couronne consacre à l'usage du rideau, à la cour des rois sassanides aussi bien qu'à celle des califes omeyyades ou abbassides, contredisent radicalement l'interprétation théologienne et symbolique classique que reçoit communément cette tradition du voile, celle que développe notamment E. Kantorowicz et qui renvoie au rideau du Tabernacle, dérobant aux yeux des hommes le Saint des Saints ${ }^{71}$. Une interpétation plus directement politique, celle qui verrait dans le voile du souverain la "manifestation" d'un lieu vide, d'une fonction politique "en creux", serait tout aussi difficile à dégager de l'argumentation de l'ouvrage ${ }^{72}$. Loin de définir un lieu vide ou un lieu sacré, le rideau désignerait au contraire aux yeux de la Cour un lieu où le souverain peut, à l'abri des égards, laisser déborder sa nature et se livrer à tous les plaisirs. Cette fonction du rideau serait d'ailleurs soulignée par le nom persan, khorem-bach, du "chambellan» préposé au rideau, qui a précisément pour signification «Sois gai» ${ }^{73}$.

Loin de signifier à la Cour le mystère du prince, le voile en souligne donc objectivement les faiblesses ${ }^{74}$ :

«J'ai demandé à Ishaq ibn Ibrahim si les Califes omayyades se montraient à leurs familiers et à leurs chanteurs. D'après lui: "Mo'awiya, Marwan Ier, 'Abd al-Malik, al-Walid Ier, Solaiman, Hicham et Marwan II ibn Mohammad étaient séparés de leurs familiers par un rideau, de sorte qu'aucun d'eux ne pouvait voir les mouvements du Calife lorsque, transporté de joie et de volupté par la musique, il se renversait, marchait, se trémoussait, dansait et se déshabillait, à un endroit où seules le voyaient les esclaves attachées à sa personne. Mais quand, derrière la tenture, s'élevait une voix, un cri de joie, un bruit de danse ou de mouvement plus fort que de raison, le préposé au rideau s'écriait: "Suffit,

70 Ed. et introd. Zeki Pacha, Le Caire, 1914. Sur la polémique concernant l'attribution de l'ouvrage, voir l'introduction de Ch. Pellat, op. cit:; Kilito, L'auteur et ses doubles, Paris, 1985, 78 sq; Schoeler, G., "Verfasser und Titel des dem Gāhiz zugeschriebenen sog. Kitāb al-Tāj», Z. D. M. G., cxxx (1980), 217-225.

71 Op. cit., Voir également du même auteur L'Empereur Frédéric II, Paris, 1987.

72 Voir Bazin, J., "Le roi sans visage»... in A visage découvert, Paris, 1992, 103-111. des.

73 Voir Kitāb, 56 sq; Mas'ūdī, Les Prairies d'Or, II, 158, Christensen, Les Sassani-

74 Cet usage du voile est destiné aux familiers du prince et non aux sujets lors des audiences ordinaires. Voir Sourdel, D., op. cit.. 
esclave! Cesse! Arrête! Assez!", pour faire croire aux courtisans que l'auteur de ce bruit était une esclave» 75 .

La fiction de la sacralisation ou simplement la convention du respect est donc dénoncée à mesure qu'elle est énoncée. Plus encore, le calife, dans cette perspective, n'est pas une figure ou une instance génériques. L'auteur du Kitāb al-Täj énumère très précisément, en se fondant classiquement sur une chaîne de témoignages oculaires, les usages du rideau propres à chacun des individus ayant accédé au califat, certains ayant d'ailleurs refusé son emploi:

«Au début de son règne, al-Mahdi se déroba à la vue de ses familiers pendant une année, pour suivre l'exemple d'al-Mançour, puis il se montra à eux. Abou-'Aun lui ayant conseillé de se cacher derrière un rideau, il répondit: «Laisse-moi donc, ignorant! Je prends plaisir au spectacle de la joie et au contact de celui qui m'égaie; quel avantage, quel plaisir y a-til en être séparé?» ${ }^{76}$.

D'autres califes ont à l'inverse recouru au rideau sur le conseil d'un courtisan:

"Au début de son règne, Abou-l-'Abbas se montrait à ses familiers, mais au bout d'une année, il se déroba à leur vue sur le conseil d'Osaid ibn 'Abd Allah al-Khoza'i. Il s'abandonnait à la joie et à la gaité et criait de derrière le rideau: "Très bien, par Dieu! Recommence cet air!"” 77.

De la même façon, les habitudes d'ivresse de tel ou tel calife, tout comme l'abstinence à cet égard de 'Umar ibn 'Abd al-'Aziz, sont scrupuleusement recensées, sans jugement moral ou religieux apparent ${ }^{78}$.

L'individualisation rigoureuse de chaque règne exclut alors, semble-t-il, que l'on puisse recourir à des interpétations globales et génériques, telle cette interprétation classique, que l'on retrouve notamment sous la plume d'O. Grabar, privilégiant la notion de transgression et définissant le droit à l'ivresse comme l'essence même du pouvoir royal ${ }^{79}$.

75 Op. cit., 59.

76 Op. cit., 63.

77 Op. cit., 61. Voir Mas'ū ${ }^{\mathrm{d}} \overline{1}$, op. cit., t. IV, 948-49.

78 'Umar b. 'Abd al-'Aziz incarne évidemment l'imitation à la lettre de l'Islam des Compagnons, des premiers califes.

79 Grabar, O., "Notes sur les cérémonies umayyades», in M. Ayalon (éd.), Studies in the memory of G. Wiet, Jérusalem, 1977; Christensen, op. cit. 
Ce que donne à voir clairement l'auteur du Kitāb al-Tāj, c'est en effet une pluralité de modèles de souveraineté possibles, fûssent-ils transgressifs par rapport à l'Islam ${ }^{80}$. Aucun système de légitimation politique n'est ici démontré de manière exclusive. Mieux encore, c'est la discordance des usages et des pratiques que fait l'objet d'une démonstration.

De manière plus générale, les signes de majesté du pouvoir souverain sont explicitement présentés dans l'ouvrage comme relevant de la convention et de la fiction, mais c'est surtout leur caractère éminemment optionnel et presque aléatoire qui fait problème ${ }^{81}$. Peut-être cet accent sur les discordances des modèles politiques, leur diffraction, estil le propre du genre littéraire des «arts de gouverner». La destination de ces ouvrages est en effet assez multiple et floue; ils combinent l'éloge des souverains et les mises en garde les plus sévères contre leur inconstance ou leur injustice. Plusieurs types de lecteurs, plusieurs destinataires, des messages contradictoires expliqueraient alors que le «mystère» soit ainsi dénoncé ou plutôt «désénoncé» à mesure qu'il est énoncé. Cependant, hors de ce genre prescriptif -qui, comme on l'a souligné, essaime et se dilue dans d'autres textes politiques- on retrouverait le même constant procédé de réduction de la sacralité politique par ceux là même qui en défendent le principe et l'efficace.

Ce problème, en effet, ne recouvre pas, ou pas seulement, les classiques débats des 'ulamā', défenseurs du strict modèle de l'imitation du Prophète, et des conseillers du prince, plus enclins à emprunter à la royauté ses techniques du gouvernement des hommes. On ne réduit pas si aisément à des "partis", ou à des "milieux" sociaux ou religieux, de telles incohérences, dès lors que c'est au sein d'un même discours que se lit la simultanéité de thèses contradictoires. Aborder plus systématiquement ces contradictions pourrait alors permettre de saisir plus justement l'essence d'une légitimité souveraine.

Une première façon d'aborder le problème de la sacralité a été, en effet, ou est toujours, fondée sur le postulat de la croyance et de l'efficace de toute idéologie souveraine. Telle est, en la schématisant à l'ex-

80 "Al-Walid 1. ${ }^{\text {er }}$ ibn 'Abd al-Malik buvait un jour sur deux; Solaiman ibn 'Abd AlMalik un jour sur trois; 'Omar II ibn 'Abd al-'Aziz ne but jamais et n'écouta jamais de musique entre le jour où il accéda au califat et celui où il mourut; Hicham s'énivrait tous les vendredis; Yazid III ibn al-Walid et al-Walid II ibn Yazid étaient adonnés à la boisson et aux plaisirs, etc...", op. cit., 171-172.

81 A l'instar de ces fictions de juristes médiévaux qui sont au fondement des "deux corps». Outre Kantorowicz, op. cit., voir Boureau, A., Le simple corps du roi. L'impossible sacralité des souverains français ( $X V^{e}-X V I I I^{e}$ siècles), Paris, 1988. 
trême, la thèse de E. Combs-Schilling, par exemple: le chérif paraît au Maroc, et sa légitimité s'impose à tous; à l'inverse, la royauté marocaine ne serait pérenne que parce qu'elle est chérifienne; le postulat de cohérence et de cohésion est dans cette étude, maximal ${ }^{82}$.

Une autre tendance, plus récente, de la recherche, a consisté au contraire à prendre en compte la diversité des interprétations possibles d'un même discours politique, ou d'une même mise en scène, en entendant "interprétation" dans son sens théâtral ou musical. P. Sanders, notamment, ou encore Ch. Klapisch, pour le cas de rituels florentins, mettent ainsi en évidence la pluralité des réceptions et des destinations d'un même message ${ }^{83}$. Mais, partant du pluriel, c'est-à-dire de groupes d'intérêts différents et concurrents, on débouche sur une forme de nécessaire consensus, et sur des frontières estompées.

Il serait bon alors de prendre aussi en compte un langage politique qui, contrairement à cette logique du modus vivendi et du consensus, ne recherche pas nécessairement l'accord, énoncé dans la simultanéité des formules politiques divergentes sans araser leur différence.

\section{LA PUBLICITÉ DES DÉBATS?}

Il y a de celà quarante ans, dans une communication consacrée au problème de la prosternation, $\mathrm{R}$. Brunschvig rappelait que les positions sur ce problème "peuvent être différentes selon qu'il s'agit de gens du peuple, de hauts dignitaires ou de poètes de cour, ou encore de ces hommes de religion -juristes qui sont les tenants officiels de l'orthodoxie» ${ }^{84}$. Brunschvig rappelait également la diversité des positions et des attitudes, sur ce problème, des différents groupes juridiques. Cet accent mis sur la variété des acteurs est assurément présent, par exemple, dans l'ouvrage récent que $\mathrm{P}$. Sanders a consacré aux rituels politiques fatimides, mais son étude met aussi en évidence, avec finesse et pertinence, la production d'une «langue moyenne», véritable lingua franca ${ }^{85}$. Sans

82 Combs-Chilling, E., Sacred Performances, Columbia U.P., 1989.

83 Sanders, op. cit.; Klapisch-Zuber, Ch., "Rituels publics et pouvoir d'Etat", in Culture et idéologie dans la genèse de l'Etat moderne, EFR, 1985, 135-144.

${ }^{84}$ Brunschvig, R., "La prosternation devant le souverain et la doctrine de l'Islam", Actes du VII Congrès international de l'histoire des religions, Florence, 1995, 438. Merci à L. Valensi pour m'avoir communiqué ce texte.

85 Op. cit. 
compétence aucune pour discuter l'histoire fatimide, je voudrais cependant m'interroger, à partir d'un exemple qui est aussi maghrébin -le parasol-, sur le degré de consensualité qui est réellement recherché dans un discours instaurant ou préconisant des formes de sacralisation des souverains.

Tout d'abord, on doit souligner que la croyance en la sacralité du prince n'est jamais assumée, revendiquée, et que les faits de croyance de ce type sont toujours attribués à autrui; ils sont plus généralement le propre de l'adversaire politique. De manière plus générale, et hors de ce registre politique, l'imputation de croyance se produit dans un rapport d'altérité ${ }^{86}$. Un exemple de ce rapport projectif serait la façon dont Tabarī décrit le culte rendu par les Shi ites -et plus précisément par le parti de Mukhtār- à un siège hérité de 'Alī, vénéré à l'instar de la $\mathrm{Ka}{ }^{\mathrm{b}} \mathrm{ba}$. Cet exemple du siège est d'autant plus fortement chargé de connotations que c'est à partir de l'adoption d'un siège de justice -que d'autres auteurs font commencer avec le règne de Mu'āwiya- que les historiens musulmans datent le processus selon lequel le califat se serait dégradé en mulk, en «royauté» ${ }^{87}$ :

"Alī avait eu un siège sur lequel il s'asseyait toujours quand il donnait des audiences de jugement. Ce siège était en la possession d'un neveu de 'Alī, nommé Tofail, qui habitait Koufa. Moukhtār fit appeler Tofail et le lui demanda, en lui promettant une récompense. Tofail dit qu'il ne l'avait pas. Comme Moukhtār insistait, Tofail demanda un délai d'une semaine, pour le retrouver; mais Moukhtār ne lui accorda que trois jours. Tofail, dans son embarras, imagina une ruse. Il acheta d'un marchand d'huile, qui demeurait dans sa rue, un siège, le fit transporter secrètement chez lui, le lava et le mit en état et le porta ensuite à Moukhtār. Celui-ci fut très heureux, récompensa généreusement Tofail, se leva, embrassa le siège et fit une prière de deux prosternations. Ensuite il dit: $\mathrm{Ce}$ siège est pour les partisans de la cause de 'Alī de que le Maqām-Ibrahìm est pour tous les musulmans, et ce qu'était pour les fils d'Israël l'arche dans laquelle se trouvaient les dix commandements de Moïse (...) Les Schi'ites touchèrent ce siège et l'embrassèrent. Moukhtār fit faire une boîte, dans laquelle il fit placer le siège; elle fut couverte d'un voile tissu

86 Voir notamment MacGaffey, W., Religion and Society in Central Africa, University of Chicago Press, 1986, 1; Pouillon, J., "Remarques sur le verbe "croire», in M. Izard et P. Smith (eds.), La Fonction symbolique, Paris, 1979; Veyne, P., Les Grecs ont-ils cru à leur mythes, Paris, 1983.

87 Cf. Ibn Khaldūn, Muqaddima. 
d'argent, confiée à des gardiens et exposée dans la mosquée principale, où les fidèles, après avoir accompli leur prière, venaient la toucher" 88 .

Outre cette forme d'idolâtrie, on retrouverait classiquement, dans ce texte, des stéréotypes de la vision des Shi ites par les Sunnites, et notamment le thème de la dissimulation et de la ruse. Cependant, même si l'on examine le point de vue le plus "intérieur" qui ait pu s'exprimer sur ce problème du "culte» dû à la présence du souverain, soit le point de vue du fameux Qāọī al-Nu'mān, on retrouve aussi l'idée que la croyance en la sacralité du souverain est le fait de ceux qui sont dans l'erreur -la canaille et la recaille dans sa terminologie- ${ }^{89}$. Sa célèbre justification de la prosternation, assimilée à la posture de la prière, repose en effet sur le principe d'une intentionnalité qui s'adresse à Dieu, et non pas à l'homme devant lequel on se prosterne. La prosternation est de surcroît considérée comme un acte de dévotion abstraite, que le Qāọi déclare préférable à un acte d'adoration physique, tel que le baisemain, pourtant parfaitement intégré alors au cérémonial califal ${ }^{90}$. Ainsi, même dans ce cas extrême, l'humaine nature du calife est rappelée, dans une apparente contradiction avec la posture que l'on entend imposer aux sujets ${ }^{91}$.

Ces contradictions ne se réduisent pas à de simples rationalisations, ou à des euphémisations; le sens des énoncés demeure d'ailleurs ouvert, virtuel, tant il est fréquent que plusieurs interprétations d'une tradition "royale" soient systématiquement accolées les unes aux autres. Les origines discordantes que les historiens ou annalistes maghrébins attribuent à l'usage sultanien du parasol, mizalla, sont à cet égard instructives.

De l'emblème du parasol, adopté par les califes fatimides, P. Sanders donne une interprétation symbolique convaincante, mais dont on ne peut s'étonner qu'elle ne soit pas formulée par les historiographes fatimides ${ }^{92}$. Sanders met en évidence un lien entre le parasol et la question de la continuité dynastique et de la succession; en cas de crise poli-

88 Les Omayyades, 89.

89 Cf. Sanders, op. cit. p. 16-17 et ss.; Brunschvig, "La prosternation...", 439.

90 Voir par exemple D. Sourdel sur la question du baise-main et Sanders, P., op. cit..

91 Sur le problème des postures corporelles et de la contracition, "L'histoire est dans l'attente...", op. cit.

92 Voir Boureau, A., "Etat moderne et attribution symbolique: emblèmes et devises dans l'Europe des $\mathrm{XVI}^{\mathrm{e}}$ et $\mathrm{XVII}^{\mathrm{e}}$ siècles", in Culture et idéologie dans la genèse de l'Etat moderne, EFR, 1985, 156-178. 
tique, l'héritier du trône est présenté à la foule sous le parasol. De plus, le parasol figurerait une sorte de "centre mobile», métonymie du palais, dans l'enceinte duquel il n'est jamais arboré ${ }^{93}$.

Cette interprétation, pourtant, un chroniqueur du $\mathrm{XII}^{\mathrm{e}}$ siècle, Ibn Hammād, au nom d'une dynastie maghrébine qui revendique l'héritage des Fatimides, la confirme et l'infirme tout à la fois ${ }^{94}$. Mentionnant l'usage du parasol sous le règne d'Abū 'l-Qāsim, il atteste en effet une forme d'association au trône liée à cet emblème. Encore ne l'exprime-til que sous une forme réaliste et non pas symbolique, décrivant en effet une effective dualité du pouvoir:

"Quand Abou-l-Qāsim montait à cheval, on portait, au dessus de sa tête, le parasol, même du vivant de son père. C'est en son nom qu'on expédiait les dépêches et les brevets de nomination; c'est à lui qu'on adressait les requêtes et qu'on renvoyait les députation" 95 .

La suite du texte développe néanmoins une interprétation fort triviale de l'emblème:

«Le cavalier qui le portait s'appelait porte-parasol (çahib al-mouhdalla), et c'était une fonction qu'on ne donnait qu'au plus digne. Le porteparasol marchait à côté du prince, pour le préserver des ardeurs du soleil, dès qu'elles commençaient à se faire sentir» 96 .

La fonction première de l'objet est donc loin d'avoir été oubliée ou minorée. Cela n'exclue pas sa métaphorisation, mais dans un registre guerrier: il est comparé à "un bouclier monté au bout d'une lance» ${ }^{97}$. Cette interprétation est à son tour évincée, ou accolée à une autre, dans un registre poétique également religieux; une autre "voix", de surcroit, se fait entendre dans le texte, celle d'un poète sévillan:

93 Cf. Sanders, Id., 25 sq notamment; voir également M. Gaudefroy-Demombynes, introduction à Al-'Umarī, op. cit.

${ }^{94}$ Ibn Hammād, Histoire des rois 'Obaïdides, éd. et trad. M. Vonderheyden, AlgerParis, 1927.

95 "Son père avait pour lui une affection, une tendrese très vive, et tirait bon augure de tout ce qu'il pouvait faire. De son côté, Abou-l-Qāsim aimait beaucoup son père, se conformant à ses ordres, et se mettant à l'affüt de ce qui pouvait lui plaire», p. 27.

96 Id., 27-28.

97 Id., 27. 
«Le poète Mohammed b. Hāni al-Andalosi a dit à ce sujet, dans une pièce composée à la louange de Ma'add al-Mo'izz, dont nous verrons bientôt l'histoire:

"Une nuée apparaît au dessus de la tête du commandeur des Croyants, elle donne à son diadème un épais ombrage."

Le parasol se dresse lentement, comme une cotte de mailles dont le tissu est renforcé; il s'étend au-dessus de lui comme de l'or fondu" ${ }^{98}$.

Il semble que les historiens des emblèmes musulmans aient été peu sensibles au fait que le motif de la nuée couvrant la tête d'un homme d'exception réfère directement au modèle de Muhammad, dont la destinée est reconnue, grâce à ce signe, par le moine chrétien Bahīra:

"Il y avait aux portes de la ville un couvent où résidait un moine nommé Bahïra, qui avait lu les anciens écrits et y avait trouvé la description du Prophète. Il y avait près de là une station où s'arrêtaient toutes les caravanes qui y passaient. La caravane d'Abū Țālib y arriva pendant la nuit. Quand le jour fut venu, laissant brouter les chameaux, les gens se mirent à dormir. Muhammad était assis et gardait leurs effets. Lorsque le soleil devint plus chaud, un nuage ayant la forme d'un grand bouclier vint ombrager la tête du Prophète. Voyant celà, le moine ouvrit la porte du couvent et en sortit: les gens de la caravane se réveillèrent" ${ }^{99}$.

Ayant interrogé Muhammad sur sa famille, Bahïra trouve ensuite entre ses épaules le sceau de la prophétie. Ce n'est donc pas une sacralité d'ordre cosmique qui est ici à l'oeuvre, mais bien un modèle islamique enraciné dans la tradition la plus canonique.

Ce constant glissement de registre se poursuit alors dans le texte d'Ibn Hammād, à travers le problème de l'attribution d'une origine à l'emblème. Un postulat d'exclusivité est en effet répété, mais pour être aussitôt démenti:

«Le parasol distinguait les 'Obaïdides de tous les autres rois» 100.

"On ne connait aucun roi, en dehors des 'Obaïdides, qui ait fait usage du parasol (Obaïd-Allah) étant devenu maître des Francs de Sicile, on

$98 \quad I d ., 28$.

99 Voir Țabarī, ed. Zotenberg, t. 2, 244-245. Pour d'autres exemples d' "hommes au nuage", voir Mas'ūdī, Les Prairies d'or, t. 1, 62 ou al-Ibshīhī, op. cit. Voir également les Mille et une Nuits (cf. Elisseeff, Thèmes et motifs des Mille et une Nuits, Beyrouth, 1949, 133).

100 Op. cit., 27. 
croit que c'est eux qui lui donnèrent le parasol, entre autres présents; c'est du moins ce que j'ai entendu dire» 101 .

Quittant le terrain de la légitimité islamique, fût-elle purement mimétique, l'auteur attribue donc à des Chrétiens l'origine de l'emblème, faisant entendre encore d'autres voix dans son récit, se faisant l'écho d'une sorte de tradition orale concurrente.

Une telle diversité de registres et d'interprétations, dans la simultanéité d'un seul énoncé, ne renvoie pas nécessairement, comme on le constate, à des groupes sociaux distincts, pas plus qu'à des genres de discours spécifiques. Elle a plutôt pour effet de souligner une forme de béance du sens, ou à l'inverse -mais ces inverses s'équivalent- un tropplein de sens. Cette même convergence de significations décalées ou même irréductibles l'une à l'autre se retrouve à l'autre extrémité du Maghreb, dans le Maroc saadien du XvI ${ }^{\mathrm{e}}$ siècle.

La métaphore du nuage, tout d'abord, est également présente sous la plume de l'historiographe al-Fishtālī:

«Les Boyyāk fournissaient (...) le porteur du parasol qui pendant la marche ombrageait la tête du sultan comme un gros nuage; ce porteur était l'officier le plus élevé en grade après le caïd Perviz' 102 .

Le cadre général de cette mise en scène èst par ailleurs sous le signe de la vertu guerrière, sur le modèle du Jihād:

«Le tout formait un ensemble tel qu'il jetait l'effroi dans les coeurs» 103

Les historiens marocains ont cependant connaissance d'autres aitiologies; l'un des prédécesseurs d'al-Manșūr, al-Ghālib billāh, aurait emprunté cet objet au saint Abū 'Ammār al-Qasțallī:

"Il gardait comme une relique un parasol en feuilles sèches de palmiers qui avait appartenu à ce saint personnage; il s'en servait pour se garantir de la chaleur, dans le but de s'attirer la "baraka" attachée à cet objet sanctissime» 104 .

101 Id., 28.

102 Cf. Nāṣirī, op. cit., p. 293.

103 Id

104 Id., 77. 
On retrouve ainsi le sens trivial du parasol, combiné avec le registre religieux de l'humilité dans la piété. Cette "invention" saadienne du parasol est d'ailleurs controversée par un autre historien, al-Ifrānī; se référant à Ibn Khallikān, il restitue pour sa part aux Fatimides la paternitè de l'emblème ${ }^{105}$.

Ces flottements attributifs et interpétatifs ne sont pas anecdotiques, pas plus qu'ils ne désignent comme accessoire leur support emblématique; ils ne se réduisent pas non plus systématiquement à autant de «versions" propres à des groupes ou des milieux sociologiquement identifiables. Aussi est-ce peut-être, au moins pour une large part, dans la référence indécidable à plusieurs histoires ou significations que se crée le sens, et dans la référence à plusieurs versions admises, en vigueur et, en un mot "consacrées".

Quel effet de sens peut alors résulter de ces juxtapositions? On retiendra en premier lieu la mise en scène de postures et d'enseignements discordants, qui peut se lire par exemple à travers le thème du sultan nourricier. L'image du sultan nourrissant ses sujets est tout d'abord un lieu commun de la littérature sultanienne, qui s'énonce, par exemple dans le Kitäb al-Tāj, dans une forme de paradoxe. Le bon souverain doit en effet couvrir largement sa table pour ses sujets et ses courtisans, qui doivent accepter pleinement ce rapport de dépendance -d'allégeance - et ne jamais apporter de nourriture au palais; à l'inverse, il est recommandé aux sujets de restreindre leur appétit et de marquer ainsi une forme de réserve ${ }^{106}$.

De manière plus générale, ce thème est formulé sur un mode métaphorique, celui du père nourrissant ses enfants ${ }^{107}$; sur un mode réaliste, et les banquets fatimides, notamment sont célèbres dans la littérature, mais aussi sur le mode de la pure fiction, comme dans ce repas purement mimétique qu'évoque P. Sanders, offert par les Fatimides à leurs sujets à l'occasion de la fête de Ghadir Khumm. En cette dernière occasion, ceux d'entre les sujets qui ne sont pas en accord avec la date officielle de rupture du jeûne sont autorisés à mimer simplement l'ingestion de nourriture. Mais indépendamment d'un tel accord consensuel, on peut mentionner une tradition tunisienne, vivace au début du $\mathrm{xx}^{\mathrm{e}}$ siècle qui veut que, lors de leur réception au palais beylical, le jour

105 Cf. Lévi-Provençal, Les Historiens des chorfas..., 127.

106 Op. cit., 39 sq.

107 Voir pour le $\mathrm{XVI}^{\mathrm{e}}$ siècle, les récits de Tamagrūtī ou Fishtālī sur les réceptions d'al-Manșūr à l'occasion du Mawlid. 
du 'Īd al-Kabīr, les invités, dans leur ensemble, se contentent de porter à leur bouche les nourritures qui leur sont offertes, avant de les reposer ${ }^{108}$. Le geste même de l'allégeance -accepter la nourriture-comporte donc son envers -elle est restituée au prince.

Ce qui est représenté est donc peut-être moins la reconnaissance du prince régnant ou de la dynastie, que celle des «lieux» où se joue le pouvoir, et des modes sur lesquels il se construit dans le cas présent par la circulation des nourritures. Il s'instaure ainsi une "scène", au double sens du lieu et de la représentation qui s'y tient, où se joue la contradiction ou l'opposition, soit, dans une certaine mesure, le débat.

Parmi d'autres exemples possibles, le problème de la sacralisation des souverains par une origine chérifienne, qui renvoie au thème de la sacralité de leur sang, illustre la simultanéité des dissonances comme l'instauration d'une scène du débat ${ }^{109}$.

On conçoit aisément qu'un témoignage extérieur, celui de voyageurs chrétiens notamment, se fasse l'écho de versions contradictoires, émises sous les murailles du palais ou dans un fondouk. La prétention des Hafsides - là encore après qu'ait été affermi leur pouvoir - à se déclarer les descendants de 'Umar b. al-Khattāb a de fait suscité de multiples contestations ou moqueries. Au XvI siècle, et selon Mármol, trois versions au moins de leur origine seraient envisageables ${ }^{110}$ :

"Ce Mulay Hascen assurait qu'il était le $35^{\mathrm{e}}$ Roye de sa famille, qui avaient régné l'un après l'autre dans Tunis, par l'espace de 450 ans et qu'ils étaient venus en droite ligne de Melchior, l'un des trois Mages. Il portait pour armes en son écu, une lance entre deux épées qui avaient la pointe en haut avec 3 croissants au dessus, et sur les croissants une couronne un estoille; ce que son fils (Muley Mahamet) me montra dans $\mathrm{Pa}$ lerme sur une épée; mais les Auteurs Africains que j’ay veus, disent que ces rois de Tunis viennent des Hentetes, qui est une branche de la tige de Muçamuda l'une des cinq principales de l'Afrique, que l'on appelle Abuhafças. D'autrent rapportent qu'ils sont descendus d'Omar second Calife, c'est pourquoy ils prennent titre d'Amir et prétendent être légitimes successeurs de Mahomet» 111

108 Cf. Samarran, Le bey de Tunis et sa cour, mémoire inédit, s.d., p. 66, IRMC, Tunis.

109 Sur la sacralité du sang, voir Bloch, M., op. cit.

110 Sur les sources de Mármol voir le travail d'édition en cours de F. R. Mediano et V. Aguilar.

111 P. 452. 
La première ascendance mentionnée, si elle n'est forgée à l'intention des Chrétiens, s'explique vraisemblablement, par l'étroitesse des liens qui unissent les princes hafsides, y compris par le sang, à des "clients» ou des alliés chrétiens ${ }^{112}$. La seconde origine renvoie à une généalogie berbère et la troisième constitue une prétention quasi "chérifienne», que renforce une éphémère revendication califale ${ }^{113}$.

Dans ce cas de figure, on peut donc admettre que le débat est sur la place publique, et que ces témoignages, directs ou indirects, reproduisent des versions qui émanent de "publics» différents ou qui visent des publics différents. Il n'est pas non plus surprenant qu'un voyageur oriental, al-'Umarī, étranger à l'Ifriqiya, ait également, dès le XIv' siècle, attesté ces débats, dans un texte, le ta'rif, repris par Qalqashandī:

«Le roi de Tunis ne prétend à rien de moins qu'au califat, prend les titres califiens, est traité d'émir el mu'minin dans son pays et affirme qu'il descend du Prince des Croyants 'Omar ben el Khattāb (qu'Allah soit satisfait de lui!). Mais des historiens instruits des généalogies nient cette descendance; les uns le font descendre des Beni 'Adi ben Kāb, branche des 'Omar, mais qui n'est pas des Beni 'Omar; d'autres disent que ce sont des Hintāta qui ne sont nullement une tribu arabe. Ce sont les Hafsides qui remontent à Aboū Hafs, l'un des dix compagnons d'Ibn Tūmert (...)»" ${ }^{114}$.

En dépit de ces dénégations à plusieurs voix, et ainsi que le remarque M. Gaudefroy-Demombynes, «bien qu'Ibn Faḍl Allāh ${ }^{115}$ refuse aux Hafsides la descendance du calife 'Omar, les formules de chancellerie de son ta'riff appellent le sultan Abū Bakr "représentant de l'imāma coréichite"»" ${ }^{116}$. Un mode de reconnaissance politique n'exclue donc pas, dans le même temps, la contestation d'une forme, non pas même de légitimité, mais de légitimation.

Or ces discussions de l'idéologie souveraine et ces confrontations de thèses caractérisent aussi les centres du pouvoir, émanent quelquefois des plus proches conseillers du prince. Il en est ainsi pour les débats concernant l'origine chérifienne des Saadiens au Maroc. Loin de

112 Voir Dufourcq, Ch. E., L'Espagne catalane et le Maghrib aux XIII et XIV siècles, Paris, PUF, 1966. Voir Adorne (Ed. Brunschvig et ed. J. Heers).

113 Cf. Brunschvig, La Berbérie..., Van Berchem, Titres califiens.

114 Cf. Qalqashandī, Șubh, t. V, 99. Voir Gaudefroy-Demombynes, M., op. cit., 99, n. 2 , et 97, n. 1 .

$115 \mathrm{Al}-{ }^{-} U$ marì. $\mathrm{V}, 134$.

116 Id. 99, n. 3. Voir également Jannabī (cf. Fagnan, Extraits inédits), Qalqashandī, 
s'en tenir à la seule version officielle de cette histoire, les historiens du temps se font aussi l'écho des contestations qui accompagnent son élaboration. Il est vrai que cette prétention s'inscrit dans le contexte d'une revendication chérifienne propre à une infinité de groupes ou de familles, y compris et surtout dans le milieu des lettrés, ministres et secrétaires.

Deux anecdotes, présentes dans différents ouvrages historiques marocains, attestent ainsi la difficulté des Saadiens, parvenus au trône, à faire reconnaître la "légitimité» de leur ascendance. La première de ces histoires met en scène un lettré, chérif, qui, à la question du sultan alMansūr: "Où nous rencontrons-nous (par la généalogie)?», répond avec insolence et humour: "Là même où nous sommes (assis)" 117 .

Cette histoire est d'ailleurs décrite par al-Ifrānī comme une aventure «que chacun sait et rapporte». Il est par ailleurs révélateur d'un rapport de forces incertain, pour le sultan, qu'al-Manșūr ait dû réprimer sa colère et se venger ultérieurement par la ruse 118 .

La seconde anecdote prête aux Saadiens, à des fins de preuve, l'exhibition de leur propre charte généalogique, authentifiée par d'illustres savants, mais des savants tunisiens, étrangers au milieu politique marocain, et qui auraient d'ailleurs "convaincu», sur ce point, le Shaykh Abū Muḥammad pré-cité. Les anecdotes elles-mêmes se répondent:

117 Voir Zayyānī, al-Ifrānī, et Nāṣirī, p. 8: «El-Mansour le Saādien était un jour assis en compagnie du pieux jurisconsulte, Abou-Mohammed Abdallah Ibn Ali, Ibn Tāher, un des chérifs de Sidjilmāssa et un homme de vertu et de religion, dans l'un de ses palais de Marrakech. Devant les deux personnages se trouvait une table à laquelle ils mangeaient. S'adressant au Cheïk Abou-Mohammed, El-Mansour lui dit: «Juriste, où sommes-nous réunis?" (il entendait par là demander à quel ancêtre commun leurs deux généalogies se confondaient). "A cette table, répondit sèchement Abou-Mohammed, ou suivant un autre récit, dans cette salle.»

118 "A ces mots, Al-Mansour fut saisi d'une violente colère, mais il la dissimula et ce ne fut que plus tard qu'il se vengea en employant un stratagème à l'aide duquel il fit perdre la vie à Abou-Mohammed.

Depuis ce jour el-Mansour invita souvent Abou-Mohammed. Malgré un froid très vif donte les rigueurs ne discontinuaient point, el-Mansour, durant ces entrevues, recevait son convive assis sur le pavé qui était de marbre. Mais il avait eu, lui, la précaution de disposer dans ses chausses un feutre de laine de façon à n'être pas incommodé par le froid. Abou-Mohammed, voyant le sultan assis comme lui sur le sol, s'armait de courage et n'osait point se lever de sa place tant que le sultan restait à discuter avec lui certaines questions scientifiques. Grâce à ce manège qui se renouvela à de nombreuses reprises, Abou-Mohammed fut pris d'un refroidissement d'entrailles; il se plaignit longemps des douleurs qu'il ressentait et qui, enfin, déterminèrent sa mort", id., p. 8. 
"El-Ifrani dit à ce sujet: "Toutefois, plusieurs de mes professeurs m’ont assuré que le Cheikh Ibn Tāher était revenu plus tard sur cette dénégation, lorsque el-Mansour lui eut montré une charte signée de l'Imām Ibn Arafa et de son professeur Ibn Abdessalām, charte qui confirmait sa généalogie. Cette circonstance calma les scrupules d'Ibn Tāher et, dans la suite, il affirma l'authenticité de cette généalogie, réprimandant tous ceux qui la révoquaient en doute» 119 .

Une telle production de preuve par le souverain constitue une véritable inversion de l'ordre politique, puisque l'une des marques de la souveraineté des souverains, marocains notamment, est précisément l'authentification des chartes chérifiennes et le contrôle des privilèges afférents ${ }^{120}$.

Que ces contestations soient, dans une forte mesure, l'écho de luttes de factions et de clans est indiscutable et les historiens font état, notamment, d'une évolution de la position idéologique des souverains alawites. Ceux-ci, après avoir contesté l'ascendance chérifienne de leurs prédécesseurs -leur concédant uniquement la nourrice du Prophète, Halima as-Sa diyya, comme ancêtre- auraient reconnu plus tardivement, une fois assuré leur propre pouvoir, un plus étroit cousinage. Les historiens contemporains, même lorsqu'ils sont rattachés au palais, rapportent avec quelque distance ces divers témoignages 121 .

$\mathrm{Au}$ bout de la chaîne des compilations, l'historien du $\mathrm{XIX}^{\mathrm{e}}$ siècle Nāșirī conclut à l'impossibilité de trancher ce problème, évacuant par là même tout système de croyance:

119 Nāșirī, 9. Voir Le Tourneau, Mélanges Louis Massignon, 1957, 65-80.

120 Voir A. Sebti, "Au Maroc: sharifisme citadin, charisme e historiographie", Annales ESC, mars-avril, 1986, pp. 433-457.

${ }^{121}$ Cf. Zayyānī, "Les Sa'diens ont fait l'objet d'attaques incessantes de la part des Chorfa du Tăfilālet qui répugnaient à être liés avec eux par l'origine comme le veut l'arbre généalogique qui vient d'être cité. Voici ce qu'en dit le Shaykh Ibn Sūda: «J'ai entendu notre shaykh Abū'l al Makkarĩ dire qu'il avait trouvé, écrite de la main de l'imām Ibn 'Arafa, la preuve authentique de leur filiation (...). Voici ce qu'en dit de son côté Abū-Zaud al-Fāsi, qu'il faut consulter avec précaution "Ce qui est pour moi établi d'après l'autorité de mon oncle Abū 'Abd Allah Muhammad b. 'Ali, qui lui-même s'appuyait sur l'autorité de son oncle Abū'-l-'Abbās, fils du shaykh Abū-l-Mahāsin, c'est qu'ils étaient descendants d'al-'Abbās b. al Muttalib: c'est à lui qu'il faisait remonter leur généalogie». D'autres ont dit qu'ils descendaient des Banū Sa'd, tribu de la nourrice du Prophète -sur lui la prière et le salut-, Halìma al-Sa'diya", in Le Tourneau, op. cit. 74, et voir pages suivantes. 
"(...) Le point sur lequel s'appuient ceux qui contestent la noblesse d'origine de ces princes consiste uniquement dans l'obscurité qui enveloppe leur arbre généalogique.

Or, il ne résulte nécessairement pas de ce manque de clarté qu'ils ne sont pas chérifs en réalité. Car, ils serait impossible, pour ceux qui leur dénient cette qualité, de connaître à fond les différentes situations de leur tableau généalogique et d'être renseignés sur tous les ancêtres qu'il comporte, depuis son commencement jusqu'à sa fin, en raison du recul considérable du temps et de la longue succession des générations. Toute recherche dans ce domaine est extrêmement difficile. C'est justement pour cette raison que le législateur musulman a laissé à chacun la latitude de s'attribuer telle ou telle origine et considère comme véridiques toutes prétentions émises en matière de généalogie qu'on ne peut connaître, le plus souvent, que par l'intermédiaire des intéressés» ${ }^{122}$.

Ces fictions de légitimation ne suscitent donc pas seulement le débat sur la place du marché ou dans le secret des salons; qu'ils soient évoqués en écho jusque par les historiens du Palais indique une forme de reconnaissance d'un espace du débat politique, hors la croyance, mais sans exclure la croyance. Ces exposés jouant de plusieurs registres et de plusieurs virtualités attestent, par ailleurs, qu'il est localement admis que ces fictions ne sont quelquefois que de purs discours d'autolégitimation, à l'usage de la Cour, ou du prince lui-même, et des discours dont l'emphase ou l'inflation varient en fonction de la conjoncture politique ${ }^{123}$. L'accord ou le consensus qui se produiraient entre souverain et sujets résulteraient moins alors de la croyance des sujets en la légitimité du prince, qu'en une reconnaissance mutuelle, un accord réciproque sur les solutions politiques les plus légitimes, acceptables, ou simplement plausibles.

122 Id. $9-10$.

${ }^{123}$ Un exemple attestant la conscience qu'il s'agit avant tout d'autolégitimation pourraît être le témoignage de Joseph de León, sous le règne de Mawlāy Ismāeîl, sur le Sacrifice du 'Ĩd al-Kabīr. La tradition marocaine, jusqu'aujourd'hui, veut en effet que l'animal égorgé publiquement par le souverain soit emporté en hâte vers le Palais; selon qu'il y arrive ou non encore en vie, on prédit pour le royaume une année faste ou néfaste. Or Joseph de Léon précise que les mulâtres chargés de transporter les bêtes sacrifiées s'arrangeaient toujours pour dire à Mawlāy Ismāeil et à la Cour qu'elles respiraient encore en arrivant du Palais, voir p. 32 et fo 71-72. 


\section{RESUMÉ}

Cet article examine la pertinence d'une distinction entre deux modes de souveraineté musulmane, sacralisée et non sacralisée, dans laquelle le Maghreb se rattacherait, dans la longue durée et de manière dominante, au deuxième modèle. Il conclut à la profonde intrication, dans la simultanéité, de différents types de légitimation du pouvoir souverain, jusque dans l'idéologie ou le discours sultaniens les plus officiels et suggère d'y voir, sinon une scène de débat, au moins une scène de la vérité des tensions et des conflits politiques.

\section{RESUMEN}

En este artículo se examina la pertinencia de distinguir entre dos formas de soberanía musulmana, sacralizada o no, dentro de las cuales el Magreb habría adoptado, en la perspectiva de "la longue durée», la segunda de estas formas. El estudio llega a la conclusión de que existe una profunda interrelación, dentro de la simultaneidad, de los diferentes tipos de legitimación del poder soberano, incluso en la ideología o el discurso del poder más oficial y sugiere que en ello debe verse, si no la existencia de debates, al menos sí la autenticidad de las tensiones y los conflictos políticos. 